\title{
Cluster cleaned HOPG by XPS
}

Morgan David J.

Citation: Surface Science Spectra 24, 024003 (2017); doi: 10.1116/1.4993771

View online: http://dx.doi.org/10.1116/1.4993771

View Table of Contents: http://avs.scitation.org/toc/sss/24/2

Published by the American Vacuum Society

\section{Instruments for Advanced Science}

Contact Hiden Analytical for further details: w www.HidenAnalytical.com E info@hiden.co.uk CLICK TO VIEW our product catalogue

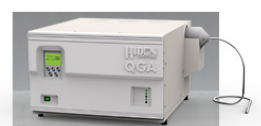

Gas Analysis

, dynamic measurement of reaction gas streams catalysis and thermal analysis

molecular beam studies

dissolved species probes

fermentation, environmental and ecological studies

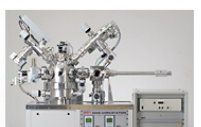

Surface Science

UHVTPD

end point detection in ion beam etch elemental imaging-surface mapping

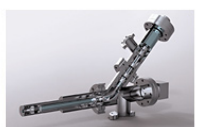

Plasma Diagnostics

' plasma source characterization

etch and deposition process reactio

, kinetic studies

analysis of neutral and radical species

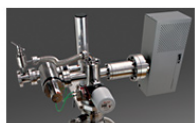

Vacuum Analysis

partial pressure measurement and contro

of process gases

, reactive sputter process control

, vacuum diagnostics

vacuum coating process monitoring 


\section{Cluster cleaned HOPG by XPS}

David J. Morgan ${ }^{\text {a) }}$

Cardiff Catalysis Institute, School of Chemistry, Cardiff University, Park Place, Cardiff CF10 3AT, United Kingdom

(Received 30 June 2017; accepted 11 September 2017; published 3 October 2017)

X-ray photoelectron spectroscopy was used to characterize a highly oriented pyrolytic graphite (HOPG) sample. The HOPG was freshly cleaved using Scotch tape prior in the entry lock of the spectrometer and subsequently cleaned using an argon cluster source. This method of cleaning has led to an improved definition of the C KLL Auger signal used for differentiation in the determination of $\mathrm{sp}^{2} / \mathrm{sp}^{3}$ ratios according to the method of Lascovich et al. [Appl. Surf. Sci. 78, 17 (1994)]. (C) 2017 American Vacuum Society. https://doi.org/10.1116/1.4993771

\begin{tabular}{l}
\hline Accession \#: 01421 \\
Technique: XPS \\
Host Material: Highly orientated \\
pyrolytic graphite \\
Instrument: Thermo Scientific K-Alpha ${ }^{+}$ \\
Major Elements in Spectra: C \\
Minor Elements in Spectra: 0 \\
Published Spectra: 4 \\
Spectra in Electronic Record: 4 \\
Spectral Category: Technical
\end{tabular}

Keywords: $H O P G, X P S$, graphite, Auger

\section{INTRODUCTION}

Highly oriented pyrolytic graphite (HOPG) is commonly used as a model carbon support for a diverse range of surface studies, including a well-defined reference material for analysis of graphite, nuclear graphite, and the functionalisation of graphitic materials (Refs. 1 and 2). Lascovich and Scaglione (Ref. 3) derived the use of the so-called D-parameter as a measure of the amount of $\mathrm{sp}^{2}$ character in a carbon material, based on the differential spectra of the C KLL Auger peak.

To date, however, the C KLL peak still shows remnants of adventitious carbon or similar as evidenced by the poor definition of the highest binding energy peak in the spectra; thus, to date the majority of x-ray induced Auger electron spectroscopy data published for HOPG is commonly that of a partially contaminated sample. The advent of cluster cleaning has allowed for a less contaminated HOPG surface to be examined.

\section{SPECIMEN DESCRIPTION (ACCESSION NO. 1421)}

Host Material: Highly orientated pyrolytic graphite (HOPG)

CAS Registry No.: 7782-42-5

Host Material Characteristics: Homogeneous; solid; single crystal; conductor; inorganic compound; other

Chemical Name: Carbon

Source: SPI Supplies

Host Composition: Carbon

Form: Crystalline wafer

Structure: C

History and Significance: Newly acquired HOPG

As Received Condition: The sample was received as new from the supplier in a sealed plastic box.

Analyzed Region: Approximately the center of the sample (of dimensions ca. $10 \times 10 \times 5 \mathrm{~mm}$ ) was analyzed with a $400-\mu \mathrm{m}$ analysis area.

Ex Situ Preparation/Mounting: The sample was placed directly on to the spectrometer sample plate and held with copper clips. The surface was cleaved with Scotch tape immediately before evacuation to UHV conditions.

a)Electronic mail: MorganDJ3@ @ardiff.ac.uk
In Situ Preparation: The sample was analyzed via XPS to ascertain any contamination from the scotch tape or similar. The sample was subsequently sputtered using a MAGCIS source operating at $4 \mathrm{kV}$ with large argon clusters of approximately 2000 atoms and rastered over an area of ca $2 \times 2 \mathrm{~mm}$.

Charge Control: No charge control was used as HOPG is a conducting sample

Temp. During Analysis: $300 \mathrm{~K}$

Pressure During Analysis: $9.33 \times 10^{-7} \mathrm{~Pa}$

Preanalysis Beam Exposure: The sample was analyzed for a total of $5 \mathrm{~min}$ prior to sputtering. Subsequent analysis after sputtering using argon clusters involved no pre-exposures.

\section{INSTRUMENT DESCRIPTION}

Manufacturer and Model: Thermo Scientific K-Alpha ${ }^{+}$

Analyzer Type: Double focussing hemispherical analyzer

Detector: Multichannel resistive plate

Number of Detector Elements: 128

INSTRUMENT PARAMETERS COMMON TO ALL SPECTRA -

\section{Spectrometer}

Analyzer Mode: Constant pass energy

Throughput $\left(T=E^{N}\right)$ : Choose an item.

Excitation Source Window: None

Excitation Source: Al Ka monochromatic

Source Energy: $1486.6 \mathrm{eV}$

Source Strength: $72 \mathrm{~W}$

Source Beam Size: $400 \times 400 \mu \mathrm{m}$

Signal Mode: Multichannel direct

- Geometry

Incident Angle: $60^{\circ}$

Source-to-Analyzer Angle: $60^{\circ}$

Emission Angle: $90^{\circ}$

Specimen Azimuthal Angle: $45^{\circ}$

Acceptance Angle from Analyzer Axis: $60^{\circ}$

Analyzer Angular Acceptance Width: $45^{\circ} \times 0^{\circ}$ 
Ion gun

Manufacturer and Model: Thermo Scientific MAGCIS

Energy: $4000 \mathrm{eV}$

Current: Enter ion beam current (-)

Current Measurement Method: Biased stage

Sputtering Species: Ar cluster

Spot Size (Unrastered): $120 \mu \mathrm{m}$

Raster Size: $4000 \times 4000 \mu \mathrm{m}$

Incident Angle: $58^{\circ}$

Polar Angle: $58^{\circ}$

Azimuthal Angle: $90^{\circ}$

Comment: Argon clusters of 2000 atoms were utilized for at a voltage of $4 \mathrm{kV}$, the current was approximately $5 \mathrm{nA}$.

\section{DATA ANALYSIS METHOD}

Energy Scale Correction: Not required

Recommended Energy Scale Shift: Not required

Peak Shape and Background Method: Peak analysis is performed using CASAXPS v2.3.19 using a Shirley background. Differentiation of the C KLL Auger peak is performed using a polynomial regression background type in CASAXPS with the first parameter set to 32 , and the differential of this data envelope performed using the Test data tab. The D-parameter is obtained using the "SP" background type, the value of which is found to be 23 in accordance with Ref. 3. A finite Lorentzian (LF) lineshape, as described by LF $(0.75,1,100,150)$, was used to model the $\mathrm{C} 1 \mathrm{~s}$ asymmetry to obtain the peak FWHM, together with a mixed Gaussian-Lorentzian peaks for the satellite structure. Details of these lineshapes can be found in Ref. 4.

Quantitation Method: Data analysis was performed in CasaXPS using a Shirley background and utilizing Scofield sensitivity factors with an energy dependence of -0.6 .

\section{ACKNOWLEDGMENTS}

The author would like to thank P. R. Davies for the loan of the HOPG sample and the Ser Cymru capital equipment grant for help in purchase of the spectrometer.

\section{REFERENCES}

1. W. Xie, K. Mo Ng, L.-T. Weng, and C.-M. Chan, RSC Adv. 6, 80649 (2016).

2. R. Burgess et al., J. Catal. 323, 10 (2015).

3. J. C. Lascovich and S. Scaglione, Appl. Surf. Sci. 78, 17 (1994).

4. S. J. Freakley, J. Ruiz-Esquius, and D. J. Morgan, Surf. Interface Anal. 49, 794 (2017). 


\section{SPECTRAL FEATURES TABLE}

\begin{tabular}{|c|c|c|c|c|c|c|c|}
\hline $\begin{array}{l}\text { Spectrum } \\
\text { ID \# }\end{array}$ & $\begin{array}{l}\text { Element/ } \\
\text { Transition }\end{array}$ & $\begin{array}{c}\text { Peak Energy } \\
(\mathrm{eV})\end{array}$ & $\begin{array}{l}\text { Peak Width } \\
\text { FWHM (eV) }\end{array}$ & $\begin{array}{c}\text { Peak Area } \\
(\mathrm{eV} \text { counts/s) }\end{array}$ & $\begin{array}{l}\text { Sensitivity } \\
\text { Factor }\end{array}$ & $\begin{array}{c}\text { Concentration } \\
\text { (at. \%) }\end{array}$ & $\begin{array}{c}\text { Peak } \\
\text { Assignment }\end{array}$ \\
\hline $1421-01$ & C 1s & 285.0 & 2.1 & $\ldots$ & 1.00 & 100 & $\mathrm{Sp}^{2}$ carbon \\
\hline 1421-01 & C KLL & 1218.0 & $\ldots$ & $\ldots$ & $\ldots$ & $\ldots$ & Carbon Auger \\
\hline 1421-02 & C 1s & 284.5 & 0.63 & 452747.3 & 1.00 & 99.9 & $S p^{2}$ carbon \\
\hline 1421-03 & $01 \mathrm{~s}$ & 532.7 & 2.0 & 1490.9 & 2.93 & 0.1 & $\begin{array}{l}\text { Oxygen bound } \\
\text { to carbon }\end{array}$ \\
\hline $1421-04$ & C KLL & 1217.9 & $\ldots$ & ... & $\ldots$ & $\ldots$ & $\begin{array}{l}\mathrm{Sp}^{2} \text { carbon } \\
\text { Auger }\end{array}$ \\
\hline
\end{tabular}




\section{ANALYZER CALIBRATION TABLE}

\begin{tabular}{lccccccc}
\hline $\begin{array}{l}\text { Spectrum } \\
\text { ID \# }\end{array}$ & $\begin{array}{c}\text { Element/ } \\
\text { Transition }\end{array}$ & $\begin{array}{c}\text { Peak Energy } \\
(\mathbf{e V})\end{array}$ & $\begin{array}{c}\text { Peak Width } \\
\text { FWHM (eV) }\end{array}$ & $\begin{array}{c}\text { Peak Area } \\
(\mathbf{e V} \text { counts/s) }\end{array}$ & $\begin{array}{c}\text { Sensitivity } \\
\text { Factor }\end{array}$ & $\begin{array}{c}\text { Concentration } \\
(\mathbf{a t .} \%)\end{array}$ & $\begin{array}{c}\text { Peak } \\
\text { Assignment }\end{array}$ \\
\hline 1 & $\mathrm{Au} 4 \mathrm{f}_{7 / 2}$ & 83.99 & 0.76 & 1597652 & 9.58 & 100 & Gold metal \\
2 & $\mathrm{Ag} 3 \mathrm{~d}_{5 / 2}$ & 368.28 & 0.58 & 1876744 & 7.38 & 100 & Silver metal \\
3 & $\mathrm{Cu} 2 \mathrm{p}_{3 / 2}$ & 932.67 & 0.83 & 2205571 & 16.73 & 100 & Copper metal \\
\hline
\end{tabular}

GUIDE TO FIGURES

\begin{tabular}{lccccc}
\hline Spectrum (Accession) \# & Spectral Region & Voltage Shift & Multiplier & Baseline & Comment \# \\
\hline $1421-01$ & Survey & 0 & 1 & 0 & $\ldots$ \\
$1421-02$ & C 1s & 0 & 1 & 0 & $\ldots$ \\
$1421-03$ & O 1s & 0 & 1 & 0 & $\ldots$ \\
$1421-04$ & C KLL & 0 & 1 & 0 & $\ldots$ \\
\hline
\end{tabular}




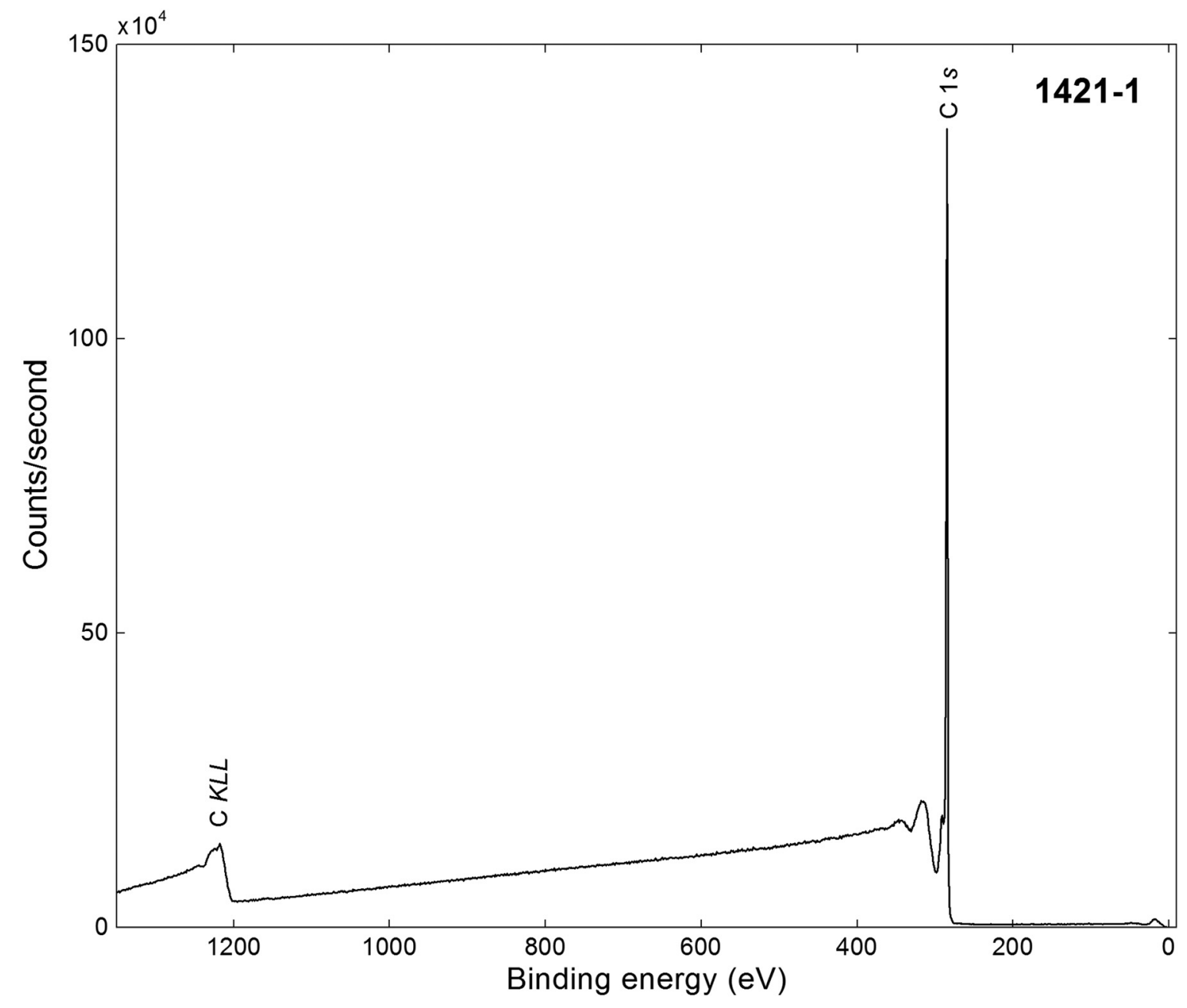

\begin{tabular}{rc}
\hline Accession \# & 1421-01 \\
Host Material & HOPG \\
Technique & XPS \\
Spectral Region & Survey \\
Instrument & Thermo Scientific K-Alpha ${ }^{+}$ \\
Excitation Source & Al Ka monochromatic \\
Source Energy & $1486.6 \mathrm{eV}$ \\
Source Strength & $72 \mathrm{~W}$ \\
Source Size & $0.004 \times 0.004 \mathrm{~mm}$ \\
Analyzer Type & $60^{\circ}$ \\
Incident Angle & $90^{\circ}$ \\
Emission Angle & $150 \mathrm{eV}$ \\
Analyzer Pass Energy & $0.1 \mathrm{eV}$ \\
Analyzer Resolution & $130 \mathrm{~s}$ \\
Total Elapsed Time & $260 \mathrm{~s}$ \\
Number of Scans & 10 \\
Total Signal Accumulation Time & $1 \mathrm{eV}$ \\
\hline
\end{tabular}




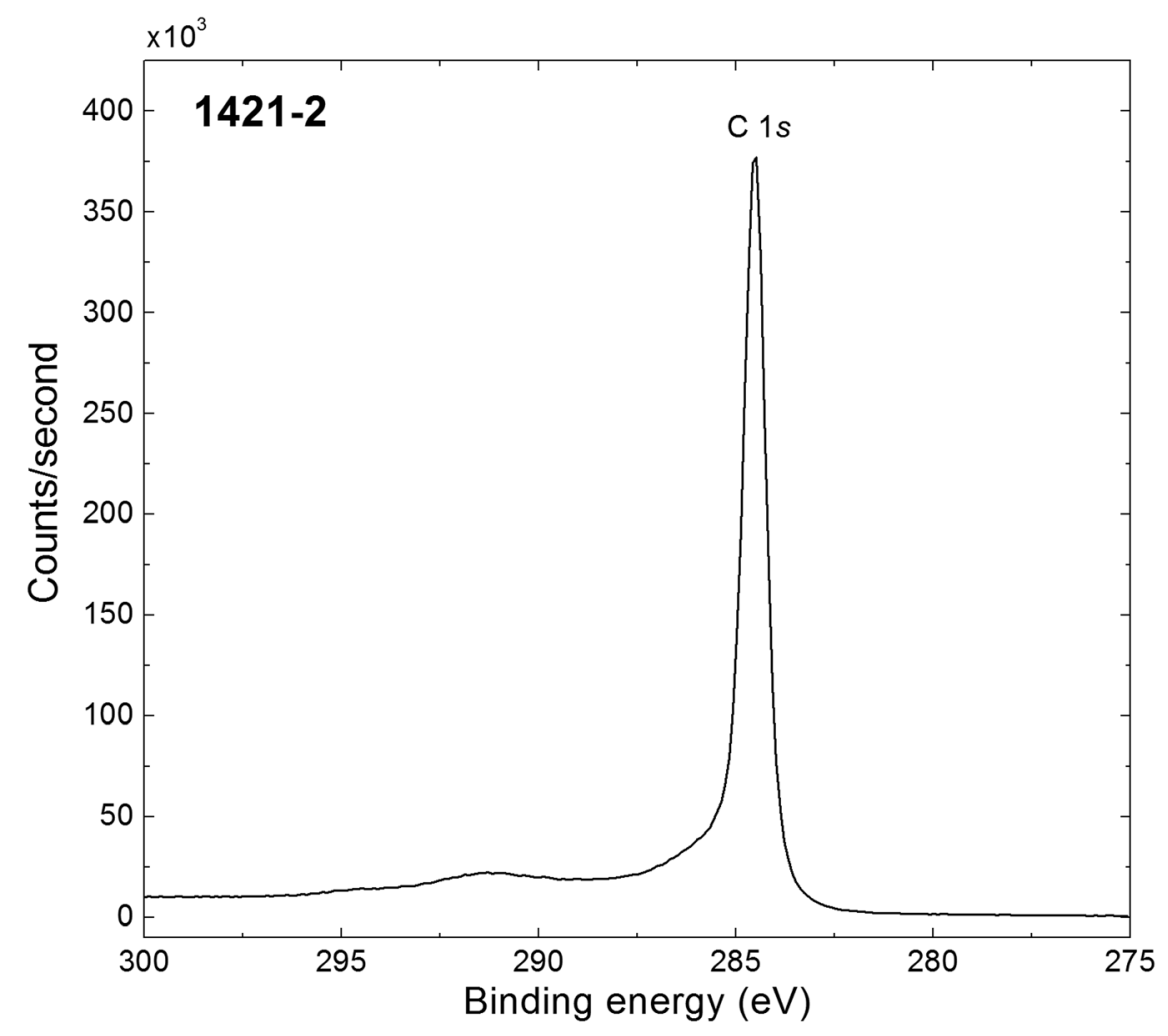

- Accession \#: 1421-02

- Host Material: HOPG

- Technique: XPS

- Spectral Region: C 1s

Instrument: Thermo Scientific K-Alpha ${ }^{+}$

Excitation Source: Al Ka monochromatic

Source Energy: $1486.6 \mathrm{eV}$

Source Strength: $72 \mathrm{~W}$

Source Size: $0.004 \times 0.004 \mathrm{~mm}$

Analyzer Type: Double focussing hemispherical analyzer

Incident Angle: $60^{\circ}$

Emission Angle: $90^{\circ}$

Analyzer Pass Energy: $40 \mathrm{eV}$

Analyzer Resolution: $0.1 \mathrm{eV}$

Total Signal Accumulation Time: $120 \mathrm{~s}$

Total Elapsed Time: $153 \mathrm{~s}$

Number of Scans: 10

Effective Detector Width: $0.1 \mathrm{eV}$

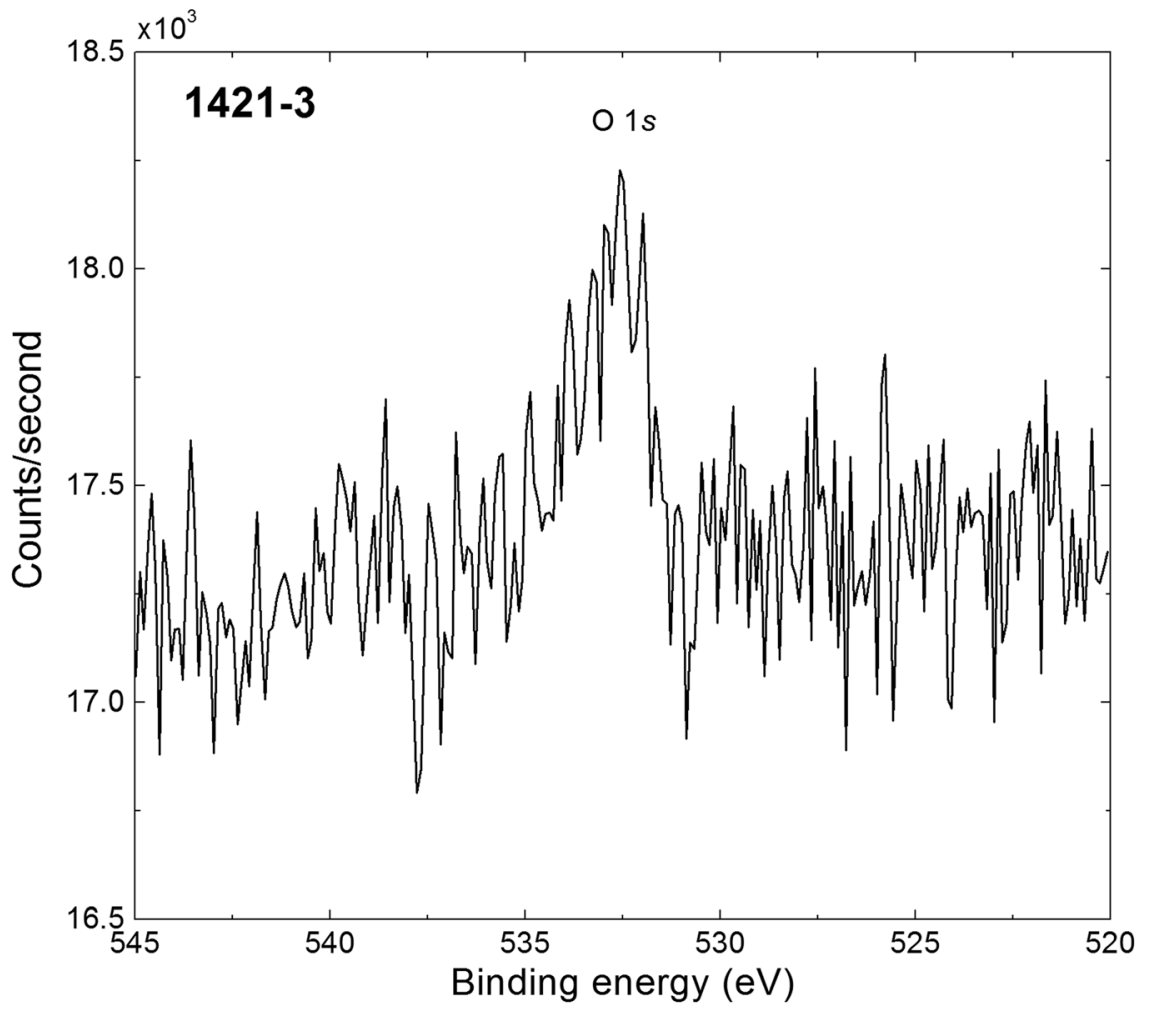

Accession \#: 1421-03

Host Material: HOPG

Technique: XPS

Spectral Region: $01 \mathrm{~s}$

Instrument: Thermo Scientific K-Alpha+

Excitation Source: Al Ka monochromatic

Source Energy: $1486.6 \mathrm{eV}$

Source Strength: $72 \mathrm{~W}$

Source Size: $0.004 \times 0.004 \mathrm{~mm}$

Analyzer Type: Double focussing

hemispherical analyzer

Incident Angle: $60^{\circ}$

Emission Angle: $90^{\circ}$

Analyzer Pass Energy: $40 \mathrm{eV}$

Analyzer Resolution: $0.1 \mathrm{eV}$

Total Signal Accumulation Time: $125 \mathrm{~s}$

Total Elapsed Time: $133 \mathrm{~s}$

Number of Scans: 10

Effective Detector Width: $0.1 \mathrm{eV}$ 


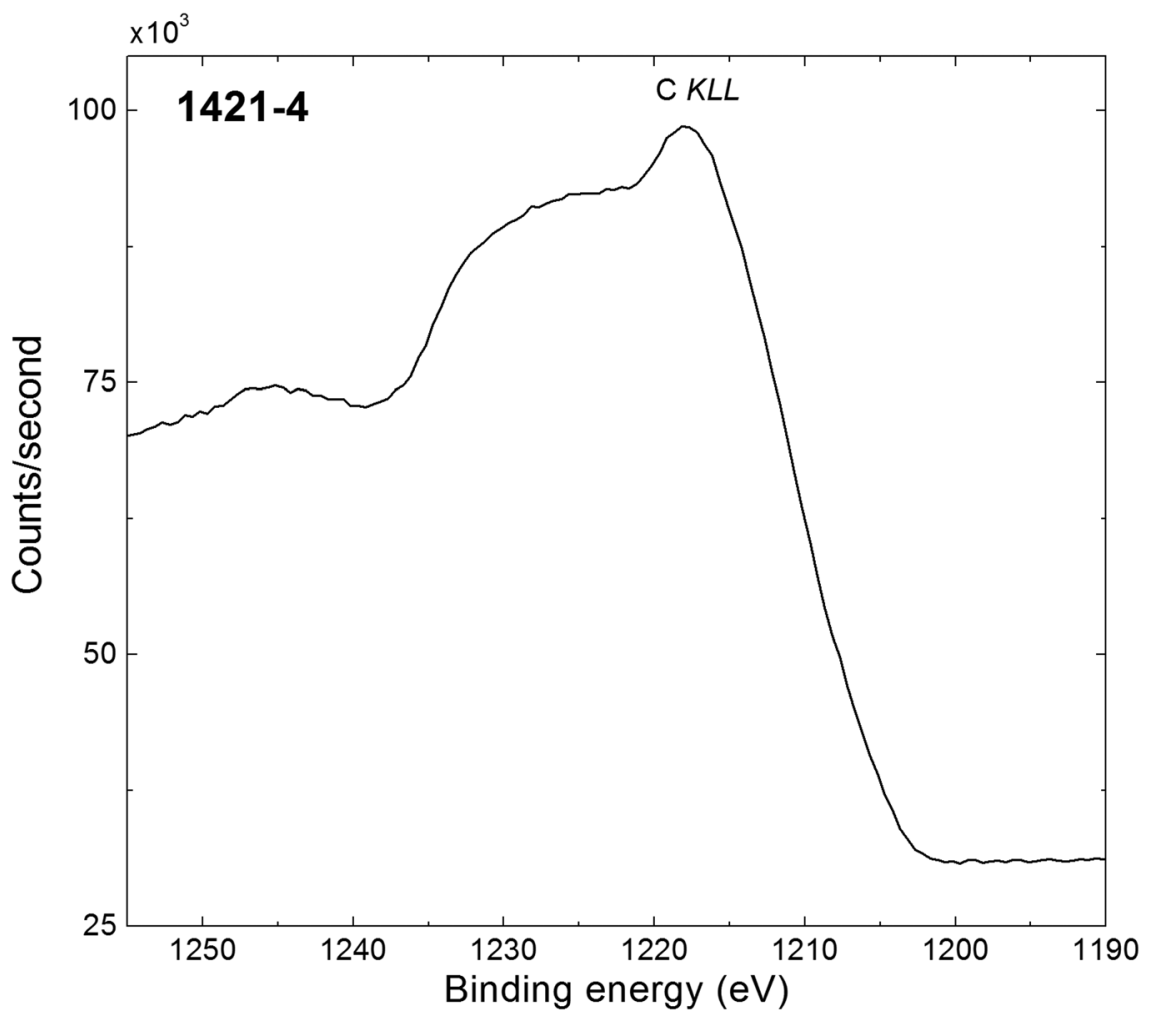

- Accession \#: 1421-04

- Host Material: HOPG

- Technique: XPS

- Spectral Region: C KLL

Instrument: Thermo Scientific K-Alpha+

Excitation Source: Al Ka monochromatic

Source Energy: $1486.6 \mathrm{eV}$

Source Strength: $72 \mathrm{~W}$

Source Size: $0.004 \times 0.004 \mathrm{~mm}$

Analyzer Type: Double focussing hemispherical analyzer

Incident Angle: $60^{\circ}$

Emission Angle: $90^{\circ}$

Analyzer Pass Energy: $100 \mathrm{eV}$

Analyzer Resolution: $0.5 \mathrm{eV}$

Total Signal Accumulation Time: $327 \mathrm{~s}$

$526 \mathrm{~s}$

Number of Scans: 50

Effective Detector Width: $0.1 \mathrm{eV}$ 